\title{
BOUNDS FOR THE DISTANCE BETWEEN THE DISTRIBUTIONS OF SUMS OF ABSOLUTELY CONTINUOUS I.I.D. CONVEX-ORDERED RANDOM VARIABLES WITH APPLICATIONS
}

\author{
TASOS C. CHRISTOFIDES, ${ }^{*}$ University of Cyprus \\ EUTICHIA VAGGELATOU, ${ }^{* *}$ University of Athens
}

\begin{abstract}
Let $X_{1}, X_{2}, \ldots$ and $Y_{1}, Y_{2}, \ldots$ be two sequences of absolutely continuous, independent and identically distributed (i.i.d.) random variables with equal means $\mathrm{E}\left(X_{i}\right)=\mathrm{E}\left(Y_{i}\right), i=$ $1,2, \ldots$ In this work we provide upper bounds for the total variation and Kolmogorov distances between the distributions of the partial sums $\sum_{i=1}^{n} X_{i}$ and $\sum_{i=1}^{n} Y_{i}$. In the case where the distributions of the $X_{i} \mathrm{~s}$ and the $Y_{i}$ s are compared with respect to the convex order, the proposed upper bounds are further refined. Finally, in order to illustrate the applicability of the results presented, we consider specific examples concerning gamma and normal approximations.
\end{abstract}

Keywords: Zolotarev's ideal metric of order 2; Kolmogorov distance; total variation distance; convex order; NBUE/NWUE; Weibull distribution; Student distribution; gamma approximation; normal approximation

2000 Mathematics Subject Classification: Primary 60E15; 62E17; 62E20

Secondary 60G50; 60K10

\section{Introduction}

Suppose that $X_{1}, X_{2}, \ldots$ is a collection of independent and identically distributed (i.i.d.) random variables and that $Y_{1}, Y_{2}, \ldots$ is another collection of i.i.d. random variables, different from the first one. It is often of interest to provide an estimate for the closeness of the distributions of the partial sums $\sum_{i=1}^{n} X_{i}$ and $\sum_{i=1}^{n} Y_{i}$ in the form of an upper bound for the total variation distance and the Kolmogorov distance, in the case where the marginal distributions of $X_{i}$ and $Y_{i}$ are compared with respect to certain classes of stochastic orderings. Such an interest also arises from the fact that the distribution of the partial sum of one of the collections is often unknown, yet the distribution of the partial sum of the other is not only known, but indeed, is one of the most frequently used in statistics for inferential purposes. For instance, it is not clear what the distribution of the partial sum of i.i.d. random variables from the Weibull distribution is. However, it is known that the Weibull distribution can be compared with the exponential distribution with respect to the so-called convex order. In addition, the sum of i.i.d. exponential random variables follows the gamma distribution. Therefore, it would be reasonable to expect that, when the difference between the variances of the marginal distributions is small,

Received 17 June 2008; revision received 9 January 2009.

* Postal address: Department of Mathematics and Statistics, University of Cyprus, P.O. Box 20537, Nicosia, CY 1678, Cyprus. Email address: tasos@ucy.ac.cy

** Postal address: Section of Statistics and Operations Research, Department of Mathematics, University of Athens, Panepistemiopolis, Athens 15784, Greece. Email address: evagel@math.uoa.gr

Partially supported by the University of Athens research grant 70/4/8810. 
the distribution of the sum of i.i.d. Weibull random variables can be well approximated by a gamma law. As demonstrated in Section 3, employing the convex order relation, upper bounds for the total variation distance and the Kolmogorov distance between the distribution of that partial sum and the distribution of the partial sum of i.i.d. exponentially distributed random variables are readily available. We would also like to emphasize that we are mainly interested in the case of fixed $n$, for otherwise, classical central limit theory provides almost all the answers regarding the closeness of the distributions of the partial sums.

In this work, our study will be focused mainly on distributions which are compared with respect to the convex order. Vaggelatou (2009) gave upper bounds for the total variation distance and the Kolmogorov distance between the distributions of two different collections of independent, integer-valued, convex-ordered random variables. For absolutely continuous random variables, in order to obtain results analogous to the discrete case, further assumptions are needed. In this paper we consider the case of absolutely continuous random variables with their partial sums having absolutely continuous densities.

In Section 2 we provide the main machinery for obtaining upper bounds. Theorem 1, which can be thought of as a 'source' result, gives upper bounds in general forms. The bounds contain quantities such as the Kolmogorov distance, the total variation distance, and the Zolotarev $\zeta_{2}$ metric between representative observations from the two collections. When comparing the distributions of the $X_{i}$ s and the $Y_{i}$ s with respect to the convex order, Corollary 1 provides upper bounds which are further refined.

In Section 3 we exploit the results of Section 2 to obtain upper bounds for the total variation distance and the Kolmogorov distance between the distributions of the partial sums of i.i.d. random variables from important specific distributions with convexly ordered marginal laws. In particular, cases considered are those of distances between the distribution of the partial sum of i.i.d. observations from the Weibull distribution and the gamma distribution. Furthermore, we consider the case of observations from more general classes of distributions, namely the so-called new better than used in expectation (NBUE) and new worse than used in expectation (NWUE) distributions. Specifically, we provide upper bounds for the case of approximating the distribution of the sum of i.i.d. NBUE and NWUE random variables by a suitable gamma distribution. Finally, we consider the case of the distribution of the partial sum of i.i.d. observations from the Student $t$-distribution and the normal distribution. The results obtained are compared with other known results in the literature.

\section{Main results}

For any two random variables $X$ and $Y$ defined on the same probability space $(\Omega, \mathrm{P})$ and with values in $\mathbb{R}$, the total variation distance between their distributions is, by definition,

$$
\rho_{\mathrm{TV}}(X, Y):=\sup _{A \in \mathcal{B}(\mathbb{R})}|\mathrm{P}(X \in A)-\mathrm{P}(Y \in A)| .
$$

In the case in which the random variables $X$ and $Y$ have densities $f_{X}$ and $f_{Y}$, respectively, the total variation distance is

$$
\rho_{\mathrm{TV}}(X, Y)=\frac{1}{2} \int_{\mathbb{R}}\left|f_{X}(t)-f_{Y}(t)\right| \mathrm{d} t .
$$

Moreover, the Kolmogorov distance is defined by

$$
\rho_{\mathrm{K}}(X, Y):=\sup _{t \in \mathbb{R}}|\mathrm{P}(X \leq t)-\mathrm{P}(Y \leq t)| .
$$


For random variables $X$ and $Y$ with finite second moments, i.e. $\mathrm{E}\left(X^{2}\right)<\infty$ and $\mathrm{E}\left(Y^{2}\right)<\infty$, the total variation distance of order 2 or Zolotarev $\zeta_{2}$-metric (see Zolotarev (1983)) is defined by

$$
\zeta_{2}(X, Y):=\int_{\mathbb{R}}\left|\mathrm{E}(X-t)_{+}-\mathrm{E}(Y-t)_{+}\right| \mathrm{d} t,
$$

where $(x)_{+}=\max (x, 0)$. Here, we note that if $X$ and $Y$ have expectations $\mathrm{E}(X)$ and $\mathrm{E}(Y)$ then the finiteness of $\zeta_{2}(X, Y)$ implies that $\mathrm{E}(X)=\mathrm{E}(Y)$, and $\zeta_{2}$ admits the following representation (see Rachev (1991, p. 258)):

$$
\begin{aligned}
\zeta_{2}(X, Y) & =\int_{\mathbb{R}}\left|\int_{-\infty}^{t}\left(F_{X}(u)-F_{Y}(u)\right) \mathrm{d} u\right| \mathrm{d} t \\
& =\int_{\mathbb{R}}\left|\int_{t}^{+\infty}\left(F_{X}(u)-F_{Y}(u)\right) \mathrm{d} u\right| \mathrm{d} t,
\end{aligned}
$$

where $F_{X}$ and $F_{Y}$ denote the cumulative distribution function (CDF) of the random variables $X$ and $Y$, respectively. It is then important to note that, from now on, whenever the distance $\zeta_{2}(X, Y)$ appears, it will be assumed that $X$ and $Y$ have finite second moments and that $\mathrm{E}(X)=$ $\mathrm{E}(Y)$. Note also that, as we will see later on, the equality of means is a natural situation that results from the convex order relation between two distributions (see Remark 2, below).

For a function $f: \mathbb{R} \rightarrow \mathbb{R}$, the symbol $f^{(k)}$ will denote the $k$ th-order derivative of $f$, i.e.

$$
f^{(k)}(x):=\frac{\mathrm{d}^{k} f(x)}{\mathrm{d}^{k} x}, \quad x \in \mathbb{R} .
$$

The result that follows offers a relationship between the Kolmogorov distance and the metric $\zeta_{2}$ for the case of real-valued random variables with densities and equal first moments.

Lemma 1. Let $X$ and $Y$ be absolutely continuous random variables with $\mathrm{E}\left(X^{2}\right)<\infty, \mathrm{E}\left(Y^{2}\right)<$ $\infty$, and $\mathrm{E}(X)=\mathrm{E}(Y)$. Also, let $Z$ be an absolutely continuous random variable independent of $X$ and $Y$. If the density $f_{Z}$ of $Z$ is an absolutely continuous function with $\left\|f_{Z}^{(1)}\right\|_{\infty}:=$ $\sup _{x \in A}\left|f_{Z}^{(1)}(x)\right|<\infty$, where $A=\left\{x \in \mathbb{R}: f_{Z}^{(1)}(x)\right.$ exists $\}$, then

$$
\rho_{\mathrm{K}}(X+Z, Y+Z) \leq\left\|f_{Z}^{(1)}\right\|_{\infty} \zeta_{2}(X, Y)
$$

Proof. Let $F_{X}$ and $F_{Y}$ denote the CDFs of $X$ and $Y$, respectively. We then have

$$
\begin{aligned}
\rho_{\mathrm{K}}(X+Z, Y+Z) & =\sup _{t \in \mathbb{R}}|\mathrm{P}(X+Z \leq t)-\mathrm{P}(Y+Z \leq t)| \\
& =\sup _{t \in \mathbb{R}}\left|\int_{\mathbb{R}}\left(F_{X}(t-z)-F_{Y}(t-z)\right) f_{Z}(z) \mathrm{d} z\right|,
\end{aligned}
$$

where the second equality follows from the independence assumption. Furthermore, using integration by parts, we derive

$$
\begin{aligned}
\int_{\mathbb{R}}\left(F_{X}(t-z)-F_{Y}(t-z)\right) f_{Z}(z) \mathrm{d} z \\
\quad=-\int_{\mathbb{R}} f_{Z}^{(1)}(z) \int_{-\infty}^{z}\left(F_{X}(t-u)-F_{Y}(t-u)\right) \mathrm{d} u \mathrm{~d} z
\end{aligned}
$$


because

$$
\begin{gathered}
\lim _{z \rightarrow \pm \infty} f_{Z}(z)=0, \\
\int_{\mathbb{R}}\left(F_{X}(t-u)-F_{Y}(t-u)\right) \mathrm{d} u=\mathrm{E}(Y)-\mathrm{E}(X)=0 .
\end{gathered}
$$

Thus, substituting (2) into (1), we finally obtain

$$
\begin{aligned}
\rho_{\mathrm{K}}(X+Z, Y+Z) & =\sup _{t \in \mathbb{R}}\left|\int_{\mathbb{R}} f_{Z}^{(1)}(z) \int_{-\infty}^{z}\left(F_{X}(t-u)-F_{Y}(t-u)\right) \mathrm{d} u \mathrm{~d} z\right| \\
& \leq \sup _{t \in \mathbb{R}} \int_{\mathbb{R}}\left|f_{Z}^{(1)}(z)\right|\left|\int_{-\infty}^{z}\left[F_{X}(t-u)-F_{Y}(t-u)\right] \mathrm{d} u\right| \mathrm{d} z \\
& \leq\left\|f_{Z}^{(1)}\right\|_{\infty} \zeta_{2}(X, Y),
\end{aligned}
$$

and the proof is complete.

Next, we prove an analogous inequality for the total variation distance.

Lemma 2. Let $X$ and $Y$ be absolutely continuous random variables with $\mathrm{E}\left(X^{2}\right)<\infty$, $\mathrm{E}\left(Y^{2}\right)<\infty$, and $\mathrm{E}(X)=\mathrm{E}(Y)$. Also, let $Z$ be an absolutely continuous random variable independent of $X$ and $Y$. If the density $f_{Z}$ of $Z$ and its derivative $f_{Z}^{(1)}$ are absolutely continuous functions then

where $\left\|f_{Z}^{(2)}\right\|_{1}:=\int_{\mathbb{R}}\left|f_{Z}^{(2)}(x)\right| \mathrm{d} x<\infty$.

$$
\rho_{\mathrm{TV}}(X+Z, Y+Z) \leq \frac{1}{2}\left\|f_{Z}^{(2)}\right\|_{1} \zeta_{2}(X, Y),
$$

Proof. If $X+Z$ and $Y+Z$ have densities $f_{X+Z}$ and $f_{Y+Z}$, respectively, then

$$
\begin{aligned}
\rho_{\mathrm{TV}}(X+Z, Y+Z) & =\frac{1}{2} \int_{\mathbb{R}}\left|f_{X+Z}(u)-f_{Y+Z}(u)\right| \mathrm{d} u \\
& =\frac{1}{2} \int_{\mathbb{R}}\left|\int_{\mathbb{R}}\left(f_{X}(t)-f_{Y}(t)\right) f_{Z}(u-t) \mathrm{d} t\right| \mathrm{d} u,
\end{aligned}
$$

where the second equality follows from the independence assumption.

Applying integration by parts twice, we obtain

$$
\begin{aligned}
\int_{\mathbb{R}}\left(f_{X}(t)-f_{Y}(t)\right) f_{Z}(u-t) \mathrm{d} t & =\int_{\mathbb{R}} f_{Z}(u-t) \mathrm{d}\left(F_{X}(t)-F_{Y}(t)\right) \\
& =-\int_{\mathbb{R}} f_{Z}^{(1)}(u-t)\left(F_{X}(t)-F_{Y}(t)\right) \mathrm{d} t \\
& =\int_{\mathbb{R}} \int_{-\infty}^{t}\left(F_{X}(s)-F_{Y}(s)\right) f_{Z}^{(2)}(u-t) \mathrm{d} s \mathrm{~d} t .
\end{aligned}
$$

Substituting (4) into (3), we obtain

$$
\begin{aligned}
\rho_{\mathrm{TV}}(X+Z, Y+Z) & =\frac{1}{2} \int_{\mathbb{R}}\left|\int_{\mathbb{R}} f_{Z}^{(2)}(u-t) \int_{-\infty}^{t}\left(F_{X}(s)-F_{Y}(s)\right) \mathrm{d} s \mathrm{~d} t\right| \mathrm{d} u \\
& \leq \frac{1}{2} \int_{\mathbb{R}} \int_{\mathbb{R}}\left|f_{Z}^{(2)}(u-t)\right|\left|\int_{-\infty}^{t}\left(F_{X}(s)-F_{Y}(s)\right) \mathrm{d} s\right| \mathrm{d} t \mathrm{~d} u \\
& =\frac{1}{2}\left\|f_{Z}^{(2)}\right\|_{1} \zeta_{2}(X, Y) .
\end{aligned}
$$

This completes the proof. 
Throughout this work, we deal with i.i.d. random variables. Therefore, in order to simplify the notation used, for any i.i.d. collection $U_{1}, U_{2}, \ldots, U_{n}$, it is convenient to set

$$
\mu_{U}:=\mathrm{E}\left(U_{i}\right), \quad \sigma_{U}^{2}:=\operatorname{var}\left(U_{i}\right), \quad i=1,2, \ldots, n,
$$

where $\mathrm{E}\left(U_{i}\right)$ and $\operatorname{var}\left(U_{i}\right)$ are the mean and variance of $U_{i}$, respectively. Furthermore, $S_{n}^{U}:=$ $\sum_{i=1}^{n} U_{i}$. In addition, for any i.i.d. collections $X_{1}, X_{2}, \ldots, X_{n}$ and $Y_{1}, Y_{2}, \ldots, Y_{n}$, we set

$$
\rho_{\mathrm{k}}:=\rho_{\mathrm{K}}\left(X_{i}, Y_{i}\right), \quad \rho_{\mathrm{tv}}:=\rho_{\mathrm{TV}}\left(X_{i}, Y_{i}\right), \quad \zeta:=\zeta_{2}\left(X_{i}, Y_{i}\right),
$$

for all $i=1,2, \ldots, n$, assuming, of course, that these quantities are positive, for otherwise, there is nothing to show.

Finally, for any density $f$, let $f_{n}$ denote the $n$th convolution of $f$, i.e.

$$
\underbrace{f * f * \cdots * f}_{n \text { times }} .
$$

We may now state the following general result.

Theorem 1. Let $X_{1}, X_{2}, \ldots, X_{n}$ and $Y_{1}, Y_{2}, \ldots, Y_{n}, n \geq 2$, be two collections where each one consists of absolutely continuous i.i.d. random variables with finite second moments. Moreover, let $f$ denote the density of either the $X_{i}$ s or $Y_{i}$ s. If $\mu_{X}=\mu_{Y}$ and the convolution $f_{n-1}$ and its derivative $f_{n-1}^{(1)}$ are absolutely continuous functions, then

$$
\rho_{\mathrm{TV}}\left(S_{n}^{X}, S_{n}^{Y}\right) \leq\left(2 \rho_{\mathrm{tv}}^{2}+2 \gamma_{\mathrm{tv}}+\frac{\gamma_{\mathrm{tv}}}{2 \rho_{\mathrm{tv}}}\right)\left(1+2 \rho_{\mathrm{tv}}\right)^{n-2}-\frac{\gamma_{\mathrm{tv}}}{2 \rho_{\mathrm{tv}}}
$$

and

$$
\rho_{\mathrm{K}}\left(S_{n}^{X}, S_{n}^{Y}\right) \leq\left(2 \rho_{\mathrm{tv}} \rho_{k}+2 \gamma_{\mathrm{k}}+\frac{\gamma_{\mathrm{k}}}{2 \rho_{\mathrm{tv}}}\right)\left(1+2 \rho_{\mathrm{tv}}\right)^{n-2}-\frac{\gamma_{\mathrm{k}}}{2 \rho_{\mathrm{tv}}},
$$

where $\gamma_{\mathrm{k}}:=\zeta\left\|f_{n-1}^{(1)}\right\|_{\infty}, \gamma_{\mathrm{tv}}:=\frac{1}{2} \zeta\left\|f_{n-1}^{(2)}\right\|_{1}, f_{n-1}^{(1)}$ and $f_{n-1}^{(2)}$ are the first and second derivatives of the density $f_{n-1}$ of the sum $\sum_{i=1, i \neq s}^{n} Y_{i}$, and $\rho_{\mathrm{k}}, \rho_{\mathrm{tv}}$, and $\zeta$ are given by (5).

In addition, if the density $f$ is bounded then, for the Kolmogorov distance, we have

$$
\rho_{\mathrm{K}}\left(S_{n}^{X}, S_{n}^{Y}\right) \leq\left(2 \rho_{\mathrm{tv}} \delta+2 \gamma_{\mathrm{k}}+\frac{\gamma_{\mathrm{k}}}{2 \rho_{\mathrm{tv}}}\right)\left(1+2 \rho_{\mathrm{tv}}\right)^{n-2}-\frac{\gamma_{\mathrm{k}}}{2 \rho_{\mathrm{tv}}},
$$

where $\delta=\min \left\{\rho_{\mathrm{tv}}, 3 M^{2 / 3} \zeta^{1 / 3}\right\}$ with $M:=\sup _{y \in \mathbb{R}} f(y)$.

Proof. Let the notation $\rho$ stand for either the Kolmogorov or the total variation distance. Using the Lindeberg decomposition, we obtain

$$
\begin{aligned}
\rho\left(\sum_{i=1}^{n} X_{i}, \sum_{i=1}^{n} Y_{i}\right) \\
\leq \rho\left(\sum_{i=1}^{n} X_{i}, \sum_{i=1}^{n-1} X_{i}+Y_{n}\right)+\rho\left(\sum_{i=1}^{n-1} X_{i}+Y_{n}, \sum_{i=1}^{n-2} X_{i}+\sum_{i=n-1}^{n} Y_{i}\right) \\
\quad+\cdots+\rho\left(\sum_{i=1}^{2} X_{i}+\sum_{i=3}^{n} Y_{i}, X_{1}+\sum_{i=2}^{n} Y_{i}\right)+\rho\left(X_{1}+\sum_{i=2}^{n} Y_{i}, \sum_{i=1}^{n} Y_{i}\right) \\
=\sum_{s=1}^{n} \rho\left(\sum_{i=1}^{s} X_{i}+\sum_{i=s+1}^{n} Y_{i}, \sum_{i=1}^{s-1} X_{i}+\sum_{i=s}^{n} Y_{i}\right) .
\end{aligned}
$$


Without loss of generality, we may assume that $Y_{1}, Y_{2}, \ldots, Y_{n}$ are independent of $X_{1}, X_{2}, \ldots$, $X_{n}$. This assumption enables us to apply the next smoothing inequality valid for $(Z, W)$ independent of $(U, V)$ :

$$
\rho(Z+U, W+U) \leq 2 \rho_{\mathrm{TV}}(Z, W) \rho(U, V)+\rho(Z+V, W+V)
$$

(cf. Proposition 2.9 of Rachev and Rüschendorf (1990) and Lemmas 14.3.2 and 17.1.7 of Rachev (1991)).

Now, applying (10) to each term in (9) with

$$
Z=X_{s}+\sum_{i=s+1}^{n} Y_{i}, \quad W=\sum_{i=s}^{n} Y_{i}, \quad U=\sum_{i=1}^{s-1} X_{i}, \quad V=\sum_{i=1}^{s-1} Y_{i},
$$

we derive

$$
\begin{aligned}
\rho\left(\sum_{i=1}^{n} X_{i}, \sum_{i=1}^{n} Y_{i}\right) \leq & 2 \sum_{s=1}^{n} \rho_{\mathrm{TV}}\left(X_{s}+\sum_{i=s+1}^{n} Y_{i}, \sum_{i=s}^{n} Y_{i}\right) \rho\left(\sum_{i=1}^{s-1} X_{i}, \sum_{i=1}^{s-1} Y_{i}\right) \\
& +\sum_{s=1}^{n} \rho\left(X_{s}+\sum_{i=1, i \neq s}^{n} Y_{i}, \sum_{i=1}^{n} Y_{i}\right) \\
= & 2 \sum_{s=2}^{n} a_{s} b_{s}+\sum_{s=1}^{n} c_{s}
\end{aligned}
$$

(the summation for $s=1$ is empty), where

$$
\begin{gathered}
a_{s}=\rho_{\mathrm{TV}}\left(X_{s}+\sum_{i=s+1}^{n} Y_{i}, \sum_{i=s}^{n} Y_{i}\right), \quad b_{s}=\rho\left(\sum_{i=1}^{s-1} X_{i}, \sum_{i=1}^{s-1} Y_{i}\right), \\
c_{s}=\rho\left(X_{s}+\sum_{i=1, i \neq s}^{n} Y_{i}, \sum_{i=1}^{n} Y_{i}\right) .
\end{gathered}
$$

Next, we will bound each $a_{s}$ and $c_{s}$ term.

By the subadditivity property of the total variation distance we obtain

$$
a_{s}=\rho_{\mathrm{TV}}\left(X_{s}+\sum_{i=s+1}^{n} Y_{i}, Y_{s}+\sum_{i=s+1}^{n} Y_{i}\right) \leq \rho_{\mathrm{TV}}\left(X_{s}, Y_{s}\right)=\rho_{\mathrm{tv}} .
$$

Furthermore, applying Lemmas 1 and 2 on $c_{s}$, we respectively derive the bounds

$$
c_{s}=\rho\left(X_{s}+\sum_{i=1, i \neq s}^{n} Y_{i}, \sum_{i=1}^{n} Y_{i}\right) \leq \begin{cases}\gamma_{\mathrm{k}} & \text { for } \rho=\rho_{\mathrm{K}} \\ \gamma_{\mathrm{tv}} & \text { for } \rho=\rho_{\mathrm{TV}}\end{cases}
$$

where

$$
\gamma_{\mathrm{k}}=\zeta\left\|f_{n-1}^{(1)}\right\|_{\infty} \quad \text { and } \quad \gamma_{\mathrm{tv}}=\frac{1}{2} \zeta\left\|f_{n-1}^{(2)}\right\|_{1} .
$$

Using inequalities (12) and (13) in (11), we obtain the following relation for the total variation distance:

$$
b_{n+1}=\rho_{\mathrm{TV}}\left(S_{n}^{X}, S_{n}^{Y}\right) \leq 2 \rho_{\mathrm{tv}} \sum_{s=2}^{n} b_{s}+n \gamma_{\mathrm{tv}} .
$$


Applying (14) for $n=2$ we can bound the quantity $b_{3}$ in terms of $b_{2}$, and by using (14) again we can bound the quantity $b_{4}$ in terms of $b_{2}$ etc.; thus, iteratively, we can bound all the $b_{s} \mathrm{~s}$ in terms of $b_{2}$, i.e. in terms of $\rho_{\mathrm{tv}}$. Having done that, standard algebraic manipulation in (14) gives the following inequality for the total variation distance:

$$
\rho_{\mathrm{TV}}\left(S_{n}^{X}, S_{n}^{Y}\right) \leq\left(2 \rho_{\mathrm{tv}}^{2}+2 \gamma_{\mathrm{tv}}+\frac{\gamma_{\mathrm{tv}}}{2 \rho_{\mathrm{tv}}}\right)\left(1+2 \rho_{\mathrm{tv}}\right)^{n-2}-\frac{\gamma_{\mathrm{tv}}}{2 \rho_{\mathrm{tv}}},
$$

i.e. inequality (6). The above inequality can be easily verified by induction.

To establish (7), we apply a similar reasoning. Now the $b_{s}$ and $c_{s}$ correspond to the Kolmogorov distance. Hence, using inequalities (12) and (13) in (11) for $\rho=\rho_{\mathrm{K}}$, we obtain the recursive inequality

$$
b_{n+1}=\rho_{\mathrm{K}}\left(S_{n}^{X}, S_{n}^{Y}\right) \leq 2 \rho_{\mathrm{tv}} \sum_{s=2}^{n} b_{s}+n \gamma_{\kappa},
$$

and the $b_{s}$ s can be found recursively in the same way as in the total variation case, finally giving

$$
\rho_{\mathrm{K}}\left(S_{n}^{X}, S_{n}^{Y}\right) \leq\left(2 \rho_{\mathrm{tv}} \rho_{k}+2 \gamma_{\mathrm{k}}+\frac{\gamma_{\mathrm{k}}}{2 \rho_{\mathrm{tv}}}\right)\left(1+2 \rho_{\mathrm{tv}}\right)^{n-2}-\frac{\gamma_{\mathrm{k}}}{2 \rho_{\mathrm{tv}}},
$$

i.e. inequality (7).

Additionally, assuming that $Y_{i}$ has a bounded density $f$, we may employ relation (14.1.16) of Rachev (1991) to obtain an upper bound for the Kolmogorov distance in terms of $\zeta_{2}$, namely,

$$
\rho_{\mathrm{k}}=\rho_{\mathrm{K}}\left(X_{s}, Y_{s}\right) \leq 3 M^{2 / 3}\left(\zeta_{2}\left(X_{s}, Y_{S}\right)\right)^{1 / 3}=3 M^{2 / 3} \zeta^{1 / 3},
$$

where $M:=\sup _{y \in \mathbb{R}} f(y)$. Using (15) and the fact that $\rho_{\mathrm{k}} \leq \rho_{\mathrm{tv}}$, we finally obtain (8).

Remark 1. For computational purposes, especially in cases where the quantity $\rho_{\mathrm{tv}}$ is not known explicitly, but rather we have a suitable upper bound for it, it would be preferable to express the bounds in (6), (7), and (8) as

$$
\begin{aligned}
& \rho_{\mathrm{TV}}\left(S_{n}^{X}, S_{n}^{Y}\right) \leq 2\left(\rho_{\mathrm{tv}}^{2}+\gamma_{\mathrm{tv}}\right)\left(1+2 \rho_{\mathrm{tv}}\right)^{n-2}+\gamma_{\mathrm{tv}} \sum_{k=1}^{n-2}\left(\begin{array}{c}
n-2 \\
k
\end{array}\right)\left(2 \rho_{\mathrm{tv}}\right)^{k-1}, \\
& \rho_{\mathrm{K}}\left(S_{n}^{X}, S_{n}^{Y}\right) \leq 2\left(\rho_{\mathrm{tv}} \rho_{\mathrm{k}}+\gamma_{\mathrm{k}}\right)\left(1+2 \rho_{\mathrm{tv}}\right)^{n-2}+\gamma_{k} \sum_{k=1}^{n-2}\left(\begin{array}{c}
n-2 \\
k
\end{array}\right)\left(2 \rho_{\mathrm{tv}}\right)^{k-1},
\end{aligned}
$$

and

$$
\rho_{\mathrm{K}}\left(S_{n}^{X}, S_{n}^{Y}\right) \leq 2\left(\rho_{\mathrm{tv}} \delta+\gamma_{\mathrm{k}}\right)\left(1+2 \rho_{\mathrm{tv}}\right)^{n-2}+\gamma_{k} \sum_{k=1}^{n-2}\left(\begin{array}{c}
n-2 \\
k
\end{array}\right)\left(2 \rho_{\mathrm{tv}}\right)^{k-1},
$$

respectively. The expressions above follow from the binomial expansion of the term $(1+$ $\left.2 \rho_{\mathrm{tv}}\right)^{n-2}$.

Next, we recall the definition of the convex order. 
Definition 1. A random variable $X$ is smaller or larger than a random variable $Y$ with respect to the convex order if $\mathrm{E} f(X) \leq \mathrm{E} f(Y)$ or, respectively, $\mathrm{E} f(X) \geq \mathrm{E} f(Y)$ for all convex functions $f$ for which the expectations exist. In such cases we write $X \preceq_{\mathrm{cx}} Y$ or $X \succeq_{\mathrm{cx}} Y$, respectively.

For more details on the properties and applications of the convex order, we refer the reader to Szekli (1995), Müller and Stoyan (2002), and Shaked and Shantikumar (2007).

Remark 2. It should be noted that the convex relation $X \preceq_{\mathrm{cx}} Y$ or $X \succeq_{\mathrm{cx}} Y$ implies that $\mathrm{E}(X)=\mathrm{E}(Y)$ and $\operatorname{var}(X) \leq \operatorname{var}(Y)$ or, respectively, $\operatorname{var}(X) \geq \operatorname{var}(Y)$. Moreover, if $X \preceq_{\mathrm{cx}} Y$ or $X \succeq_{\mathrm{cx}} Y$ then $X=_{\mathrm{st}} Y$ if and only if $\operatorname{var}(X)=\operatorname{var}(Y)$. Therefore, the closeness of the distributions of $X$ and $Y$ can be measured in terms of the difference between their variances.

Assuming that the distributions of the $X_{i}$ s and $Y_{i}$ s are compared with respect to the convex order as defined above, we can obtain upper bounds for the total variation and Kolmogorov distances in terms of the difference between their variances, $\left|\sigma_{Y}^{2}-\sigma_{X}^{2}\right|$. This is due to the fact that, for random variables $X$ and $Y$ such that $X \preceq_{\mathrm{cx}} Y$ or $X \succeq_{\mathrm{cx}} Y$, Kaas (1993) proved that the metric $\zeta_{2}(X, Y)$ is equal to

$$
\zeta_{2}(X, Y)=\frac{\left|\sigma_{Y}^{2}-\sigma_{X}^{2}\right|}{2} .
$$

Thus, we have the following result, the proof of which is immediate.

Corollary 1. Let $X_{1}, X_{2}, \ldots, X_{n}$ and $Y_{1}, Y_{2}, \ldots, Y_{n}, n \geq 2$, be two collections where each one consists of absolutely continuous i.i.d. random variables with finite second moments. Moreover, let $f$ denote the density of either the $X_{i}$ s or $Y_{i} s$. If

$$
X_{i} \preceq_{\mathrm{cx}} Y_{i} \text { or } X_{i} \succeq_{\mathrm{cx}} Y_{i} \text { for all } i=1,2, \ldots, n,
$$

and the convolution $f_{n-1}$ and its derivative $f_{n-1}^{(1)}$ are absolutely continuous functions, then inequalities (6), (7), (8), (16), (17), and (18) are valid with

$$
\zeta=\frac{1}{2}\left|\sigma_{Y}^{2}-\sigma_{X}^{2}\right|, \quad \gamma_{\mathrm{tv}}=\frac{1}{4}\left|\sigma_{Y}^{2}-\sigma_{X}^{2}\right|\left\|f_{n-1}^{(2)}\right\|_{1}, \quad \text { and } \quad \gamma_{\mathrm{k}}=\frac{1}{2}\left|\sigma_{Y}^{2}-\sigma_{X}^{2}\right|\left\|f_{n-1}^{(1)}\right\|_{\infty} .
$$

The bounds of Theorem 1 and Corollary 1 are expressed in terms of the total variation distance $\rho_{\mathrm{tv}}$ between the distributions of the coordinates $X_{i}$ and $Y_{i}$. Unfortunately, an analogue of the inequality in (15) (cf. proof of Theorem 1) for the total variation distance does not hold true. This is, in fact, a general problem for the continuous distributions. In the discrete case, the total variation distance is always upper bounded by twice the Zolotarev's metric $\zeta_{2}$, i.e. $\rho_{\mathrm{TV}}(X, Y) \leq 2 \zeta_{2}(X, Y)$ for $X$ and $Y$ taking values in $\mathbb{Z}$ (cf. Lemma 1 of Boutsikas and Vaggelatou (2008)). However, for real-valued random variables with density, $\rho_{\mathrm{TV}}$ cannot be compared with $\zeta_{2}$ (in particular, there are cases where $\zeta_{2} \rightarrow 0$ while $\rho_{\mathrm{TV}}$ remains constant). Therefore, the distance $\rho_{\mathrm{tv}}$ must be evaluated or upper bounded individually according to the distributions involved each time in the problem of interest. The latter is a situation that usually occurs in the continuous-distribution approximation error estimates (for example, in the BerryEsseen-type results).

\section{Applications}

In this section we illustrate the previous results with specific applications concerning gamma and normal approximations. 


\subsection{Distance between the sum of i.i.d. Weibull-distributed random variables and a gamma-distributed random variable}

Let $X_{1}, X_{2}, \ldots, X_{n}$ be i.i.d. random variables following the Weibull distribution $\mathcal{W}(\alpha, \theta)$ with density

$$
h(x)=\alpha \theta(\theta x)^{\alpha-1} \exp \left\{-(\theta x)^{\alpha}\right\}, \quad x>0, \alpha>0, \theta>0
$$

( $\alpha$ and $\theta$ are the shape and scale parameters, respectively). The mean and variance of $\mathcal{W}(\alpha, \theta)$ are given by

$$
\mu_{X}=\frac{1}{\theta} \Gamma\left(1+\frac{1}{\alpha}\right), \quad \sigma_{X}^{2}=\frac{1}{\theta^{2}} \Gamma\left(1+\frac{2}{\alpha}\right)-\mu_{X}^{2},
$$

where $\Gamma(\cdot)$ denotes the gamma function, i.e. $\Gamma(\beta)=\int_{0}^{\infty} t^{\beta-1} \mathrm{e}^{-t} \mathrm{~d} t, \beta>0$. We also consider a collection of i.i.d. random variables $Y_{1}, Y_{2}, \ldots, Y_{n}$ distributed according to the exponential distribution $\mathscr{E}\left(1 / \mu_{X}\right)$ with parameter $1 / \mu_{X}$, i.e. with density

$$
g(x)=\frac{\theta}{\Gamma(1+1 / \alpha)} \exp \left\{-\frac{\theta}{\Gamma(1+1 / \alpha)} x\right\}, \quad x>0 .
$$

It is known that $\mathcal{W}(\alpha, \theta) \leq_{\mathrm{cx}} \mathscr{E}\left(1 / \mu_{X}\right)$ for $\alpha>1, \mathcal{W}(\alpha, \theta) \geq_{\mathrm{cx}} \mathcal{E}\left(1 / \mu_{X}\right)$ for $\alpha<1$, and, clearly, $\mathcal{W}(\alpha, \theta)=_{\text {st }} \mathscr{E}\left(1 / \mu_{X}\right)$ for $\alpha=1$.

The distribution of the sum $S_{n}^{X}=\sum_{i=1}^{n} X_{i}$ is unknown and, therefore, it would be useful to approximate it by a known distribution. Of course, for $n \rightarrow \infty$, employing the central limit theorem we may use the normal approximation. However, we note that, when $\alpha \rightarrow 1$, $\mathcal{W}(\alpha, \theta)$ gets closer to the exponential distribution $\mathscr{E}(\theta)$ and, therefore, it is expected that, for fixed $n$ and $\alpha \rightarrow 1$, approximation by a gamma distribution would be a more appropriate solution. Here, we observe that the distribution of the $\operatorname{sum} S_{n}^{Y}=\sum_{i=1}^{n} Y_{i}$ is indeed the gamma distribution with parameters $n$ and $1 / \mu_{X}$ (denoted by $g\left(n, 1 / \mu_{X}\right)$ ), and, thus, exploiting the results of Corollary 1 , we may obtain error estimates for the distances $\rho_{\mathrm{K}}\left(S_{n}^{X}, g\left(n, 1 / \mu_{X}\right)\right)$ and $\rho_{\mathrm{TV}}\left(S_{n}^{X}, g\left(n, 1 / \mu_{X}\right)\right)$ for fixed values of $n$.

First of all we need to find the norms $\left\|f^{(1)}\right\|_{\infty}$ and $\left\|f^{(2)}\right\|_{1}$ of the density $f$ relative to the gamma distribution. Tedious calculations lead to the following general result.

Lemma 3. Let $f$ denote the density of the gamma distribution $g(v, \lambda)$, i.e.

$$
f(x)=\frac{\lambda^{\nu}}{\Gamma(\nu)} x^{\nu-1} \mathrm{e}^{-\lambda x}, \quad x>0, v>0, \lambda>0 .
$$

Then, for $v \geq 3$,

$$
\left\|f^{(1)}\right\|_{\infty}=\frac{\lambda^{2} \sqrt{v-1}}{(v-1) !} b_{v}^{\nu-2} \exp \left\{-b_{v}\right\}
$$

and

$$
\left\|f^{(2)}\right\|_{1}=\frac{2 \lambda^{2} \sqrt{v-1}}{(\nu-1) !}\left(b_{v}^{\nu-2} \exp \left\{-b_{\nu}\right\}+c_{\nu}^{\nu-2} \exp \left\{-c_{\nu}\right\}\right),
$$

where

$$
b_{v}:=v-1-\sqrt{v-1}, \quad c_{v}:=v-1+\sqrt{v-1} .
$$

We will apply Corollary 1 to obtain the desired error bounds. We have

$$
\left|\sigma_{Y}^{2}-\sigma_{X}^{2}\right|=\left|2 \mu_{X}^{2}-\frac{1}{\theta^{2}} \Gamma\left(1+\frac{2}{\alpha}\right)\right|=2 \mu_{X}^{2}\left|1-\frac{\Gamma(1+2 / \alpha)}{2 \Gamma^{2}(1+1 / \alpha)}\right| .
$$


Furthermore, we need to evaluate the distances $\rho_{\mathrm{tv}}=\rho_{\mathrm{TV}}\left(\mathcal{W}(\alpha, \theta), \mathcal{E}\left(1 / \mu_{X}\right)\right)$ and $\rho_{\mathrm{k}}=$ $\rho_{\mathrm{K}}\left(\mathcal{W}(\alpha, \theta), \mathcal{E}\left(1 / \mu_{X}\right)\right)$. Using (19) and (20), we obtain

$$
\begin{aligned}
\rho_{\mathrm{TV}}\left(\mathcal{W}(\alpha, \theta), \mathcal{E}\left(\frac{1}{\mu_{X}}\right)\right)= & \frac{1}{2} \int_{0}^{\infty}|h(x)-g(x)| \mathrm{d} x \\
= & \frac{1}{2} \int_{0}^{r_{1}}(g(x)-h(x)) \mathrm{d} x+\frac{1}{2} \int_{r_{1}}^{r_{2}}(h(x)-g(x)) \mathrm{d} x \\
& +\frac{1}{2} \int_{r_{2}}^{\infty}(g(x)-h(x)) \mathrm{d} x \\
= & \exp \left\{-\frac{\theta r_{2}}{\Gamma(1+1 / \alpha)}\right\}-\exp \left\{-\frac{\theta r_{1}}{\Gamma(1+1 / \alpha)}\right\} \\
& +\exp \left\{-\left(\theta r_{1}\right)^{\alpha}\right\}-\exp \left\{-\left(\theta r_{2}\right)^{\alpha}\right\},
\end{aligned}
$$

where $r_{1}$ and $r_{2}$ are the roots of the nonlinear equation

$$
(\theta x)^{\alpha}-\frac{\theta x}{\Gamma(1+1 / \alpha)}=(\alpha-1) \log x+\log \left(\alpha \theta^{\alpha-1} \Gamma\left(1+\frac{1}{\alpha}\right)\right)
$$

with respect to $x$. In addition, using standard calculus, it can be verified that

$$
\rho_{\mathrm{k}}=\max \left\{\varphi\left(r_{1}\right), \varphi\left(r_{2}\right)\right\}
$$

where

$$
\varphi(x):=\left|\exp \left\{-\frac{\theta x}{\Gamma(1+1 / \alpha)}\right\}-\exp \left\{-(\theta x)^{\alpha}\right\}\right| .
$$

So, finally, applying Corollary 1 and using (21) and Lemma 3, we immediately derive the following error bounds.

Proposition 1. Let $X_{1}, X_{2}, \ldots, X_{n}$ be i.i.d. observations from the Weibull distribution with density (19). For $n \geq 4$,

$$
\rho_{\mathrm{TV}}\left(S_{n}^{X}, \mathcal{g}\left(n, \frac{1}{\mu_{X}}\right)\right) \leq B_{\mathrm{TV}}^{g}:=\left(2 \rho_{\mathrm{tv}}^{2}+2 \gamma_{\mathrm{tv}}+\frac{\gamma_{\mathrm{tv}}}{2 \rho_{\mathrm{tv}}}\right)\left(1+2 \rho_{\mathrm{tv}}\right)^{n-2}-\frac{\gamma_{\mathrm{tv}}}{2 \rho_{\mathrm{tv}}}
$$

and

$$
\rho_{\mathrm{K}}\left(S_{n}^{X}, g\left(n, \frac{1}{\mu_{X}}\right)\right) \leq B_{\mathrm{K}}^{g}:=\left(2 \rho_{\mathrm{tv}} \rho_{\mathrm{k}}+2 \gamma_{\mathrm{k}}+\frac{\gamma_{k}}{2 \rho_{\mathrm{tv}}}\right)\left(1+2 \rho_{\mathrm{tv}}\right)^{n-2}-\frac{\gamma_{\mathrm{k}}}{2 \rho_{\mathrm{tv}}},
$$

where

$$
\begin{gathered}
\gamma_{\mathrm{tv}}=\frac{\sqrt{n-2}}{(n-2) !}\left(b_{n-1}^{n-3} \exp \left\{-b_{n-1}\right\}+c_{n-1}^{n-3} \exp \left\{-c_{n-1}\right\}\right)\left|1-\frac{\Gamma(1+2 / \alpha)}{2 \Gamma^{2}(1+1 / \alpha)}\right|, \\
\gamma_{\mathrm{k}}=\frac{\sqrt{n-2}}{(n-2) !} b_{n-1}^{n-3} \exp \left\{-b_{n-1}\right\}\left|1-\frac{\Gamma(1+2 / \alpha)}{2 \Gamma^{2}(1+1 / \alpha)}\right|, \\
b_{n-1}=n-2-\sqrt{n-2}, \quad c_{n-1}:=n-2+\sqrt{n-2},
\end{gathered}
$$

and $\rho_{\mathrm{tv}}$ and $\rho_{\mathrm{k}}$ are given by (22) and (23), respectively.

Remark 3. Using mathematical packages, we can easily compute the exact values of $\rho_{\mathrm{tv}}$ and $\rho_{\mathrm{k}}$. 
TABLE 1.

\begin{tabular}{ccccccccc}
\hline & \multicolumn{3}{c}{$n=5$} & \multicolumn{4}{c}{$n=10$} & \multicolumn{4}{c}{$n=20$} \\
\hline$\alpha$ & $B_{\mathrm{K}}^{g}$ & $B_{\mathrm{TV}}^{g}$ & $\alpha$ & $B_{\mathrm{K}}^{g}$ & $B_{\mathrm{TV}}^{g}$ & $\alpha$ & $B_{\mathrm{K}}^{g}$ & $B_{\mathrm{TV}}^{g}$ \\
\hline 0.90 & 0.098077 & 0.139942 & 0.90 & 0.081772 & 0.129262 & 0.90 & 0.127408 & 0.210300 \\
0.95 & 0.039789 & 0.056967 & 0.95 & 0.027896 & 0.044639 & 0.95 & 0.030055 & 0.050972 \\
0.97 & 0.022029 & 0.031578 & 0.97 & 0.014502 & 0.023306 & 0.97 & 0.013784 & 0.023578 \\
0.99 & 0.006788 & 0.009741 & 0.99 & 0.004211 & 0.006795 & 0.99 & 0.003579 & 0.006166 \\
1.01 & 0.006567 & 0.009425 & 1.01 & 0.004074 & 0.006573 & 1.01 & 0.003460 & 0.005961 \\
1.03 & 0.019932 & 0.028575 & 1.03 & 0.013092 & 0.021043 & 1.03 & 0.012381 & 0.021182 \\
1.05 & 0.033625 & 0.048157 & 1.05 & 0.023423 & 0.037496 & 1.05 & 0.024863 & 0.042189 \\
1.10 & 0.069440 & 0.099209 & 1.10 & 0.056323 & 0.089198 & 1.10 & 0.082025 & 0.135868 \\
1.15 & 0.107745 & 0.153609 & 1.15 & 0.102214 & 0.160120 & 1.15 & 0.208963 & 0.337398 \\
\hline
\end{tabular}

Remark 4. For fixed $n$ and $\theta$, when $\alpha \rightarrow 1$, the aforementioned error estimates tend to 0 , as expected.

In Table 1 , numerical values for the bounds $B_{\mathrm{TV}}^{g}$ and $B_{\mathrm{K}}^{g}$ of Proposition 1 are presented for several values of $\alpha$ and $n$. The bounds are not affected by the value of $\theta$ because the distances $\rho_{\mathrm{k}}$ and $\rho_{\mathrm{tv}}$ are stable under scale changes and, thus, for convenience, we chose $\theta=1$. Obviously, we can see that, for fixed $n$, the numerical error estimates tend to 0 when $\alpha \rightarrow 1$, as expected. The bounds were computed using MATHEMATICA ${ }^{\circledR}$ software. We also note that, for the calculations, the exact numerical values of the distances $\rho_{\mathrm{tv}}$ and $\rho_{\mathrm{k}}$ were used.

\subsection{Distance between sums of NBUE or NWUE i.i.d. observations and a gamma random variable}

The Weibull distribution considered in Subsection 3.1 belongs to specific classes of distributions provided by the following definition (cf. Barlow and Proschan (1975)).

Definition 2. The nonnegative random variable $X$ (and its distribution function $F$ ) is said to be NBUE if, for all $t \geq 0$,

$$
\frac{\int_{t}^{\infty} \bar{F}(x) \mathrm{d} x}{\bar{F}(t)} \leq \mathrm{E}(X),
$$

and it is said to be NWUE if, for all $t \geq 0$,

$$
\frac{\int_{t}^{\infty} \bar{F}(x) \mathrm{d} x}{\bar{F}(t)} \geq \mathrm{E}(X),
$$

where $\bar{F}(x)=1-F(x)$.

We can verify that the Weibull distribution is NWUE if $\alpha<1$ and NBUE for $\alpha>1$.

The following result (see Theorem 3.A.55 of Shaked and Shanthikumar (2007)) is relevant.

Theorem 2. If $X$ is an NBUE or NWUE random variable with mean $\mu$ and $Y$ is an exponentially distributed random variable with parameter $1 / \mu$, then

$$
X \preceq_{\mathrm{cx}} Y \quad \text { or, respectively, } \quad X \succeq_{\mathrm{cx}} Y \text {. }
$$


Assume now that $X_{1}, X_{2}, \ldots, X_{n}$ are i.i.d. observations from a distribution which is NBUE (or NWUE). Let $\mu_{X}$ and $\sigma_{X}^{2}$ denote the mean and variance, respectively, of the distribution. Assume further that $Y_{1}, Y_{2}, \ldots, Y_{n}$ are i.i.d. observations from the exponential distribution with parameter $1 / \mu_{X}$. We can easily verify that

$$
\left|\sigma_{Y}^{2}-\sigma_{X}^{2}\right|=\mu_{X}^{2}\left|1-C_{X}^{2}\right|,
$$

where $C_{X}=\sigma_{X} / \mu_{X}$ denotes the coefficient of variation of the random variable $X$.

In what follows, for notational simplicity, we set

$$
\begin{gathered}
A_{X}:=\frac{1}{2}\left|1-C_{X}^{2}\right|, \\
m_{n}:=\frac{\sqrt{n-2}}{(n-2) !} b_{n-1}^{n-3} \exp \left\{-b_{n-1}\right\}, \\
h_{n}:=\frac{\sqrt{n-2}}{(n-2) !}\left(b_{n-1}^{n-3} \exp \left\{-b_{n-1}\right\}+c_{n-1}^{n-3} \exp \left\{-c_{n-1}\right\}\right),
\end{gathered}
$$

where $b_{n-1}=n-2-\sqrt{n-2}$ and $c_{n-1}=n-2+\sqrt{n-2}$ (recall Lemma 3 ).

In view of (24) and Lemma 3, Corollary 1 provides the following gamma approximation bounds for $n \geq 4$ :

$$
\begin{gathered}
\rho_{\mathrm{TV}}\left(S_{n}^{X}, g\left(n, \frac{1}{\mu_{X}}\right)\right) \leq\left(2 \rho_{\mathrm{tv}}^{2}+2 h_{n} A_{X}+\frac{h_{n} A_{X}}{2 \rho_{\mathrm{tv}}}\right)\left(1+2 \rho_{\mathrm{tv}}\right)^{n-2}-\frac{h_{n} A_{X}}{2 \rho_{\mathrm{tv}}} \\
\rho_{\mathrm{K}}\left(S_{n}^{X}, g\left(n, \frac{1}{\mu_{X}}\right)\right) \leq\left(2 \rho_{\mathrm{tv}} \rho_{\mathrm{k}}+2 m_{n} A_{X}+\frac{m_{n} A_{X}}{2 \rho_{\mathrm{tv}}}\right)\left(1+2 \rho_{\mathrm{tv}}\right)^{n-2}-\frac{m_{n} A_{X}}{2 \rho_{\mathrm{tv}}},
\end{gathered}
$$

where $A_{X}, h_{n}$, and $m_{n}$ are given by (25), (26), and (27), respectively.

The upper bounds for both distances in (28) and (29) are expressed in terms of the total variation distance $\rho_{\mathrm{tv}}$ and the Kolmogorov distance $\rho_{\mathrm{k}}$. We may use a similar approach to the one described in Subsection 3.1 in order to determine the exact values of $\rho_{\mathrm{tv}}$ and $\rho_{\mathrm{k}}$. However, for NBUE or NWUE random variables, upper bounds for $\rho_{\mathrm{k}}$ were obtained in Daley (1988, Theorems 1 and 2). In particular, Daley showed that if $X$ is NBUE and $Y$ is exponentially distributed with the same mean, then

$$
\rho_{\mathrm{K}}(X, Y) \leq 1-\exp \left\{-\sqrt{2 A_{X}}\right\}
$$

whereas, if $X$ is NWUE,

$$
\rho_{\mathrm{K}}(X, Y) \leq \sqrt{A_{X}^{2}+2 A_{X}}-A_{X}
$$

Furthermore, as the following result shows, the total variation distance $\rho_{\mathrm{tv}}$ can be upper bounded by twice the quantities on the right-hand sides of (30) and (31) involving the coefficient of variation of $X$.

Lemma 4. Let $X$ be a random variable with mean $\mu_{X}$ and finite variance $\sigma_{X}^{2}$. Also, let $Y$ be an exponential random variable with parameter $1 / \mu_{X}$. If $X$ is NBUE then

$$
\rho_{\mathrm{TV}}(X, Y) \leq 2\left(1-\exp \left\{-\sqrt{2 A_{X}}\right\}\right)
$$

and if $X$ is NWUE then

$$
\rho_{\mathrm{TV}}(X, Y) \leq 2\left(\sqrt{A_{X}^{2}+2 A_{X}}-A_{X}\right)
$$


Proof. Let $h$ and $g$ denote the densities, and let $H$ and $G$ denote the CDFs of the random variables $X$ and $Y$, respectively. Since $X$ is NBUE or NWUE, by Theorem 2, the distributions of $X$ and $Y$ are ordered according to the convex order, and, therefore, their densities cross each other at exactly two points, say $r_{1}$ and $r_{2}$. Then,

$$
\begin{aligned}
\rho_{\mathrm{TV}}(X, Y)= & \frac{1}{2} \int_{0}^{\infty}|h(x)-g(x)| \mathrm{d} x \\
= & \frac{1}{2} \int_{0}^{r_{1}}(g(x)-h(x)) \mathrm{d} x+\frac{1}{2} \int_{r_{1}}^{r_{2}}(h(x)-g(x)) \mathrm{d} x \\
& +\frac{1}{2} \int_{r_{2}}^{\infty}(g(x)-h(x)) \mathrm{d} x \\
= & G\left(r_{1}\right)-G\left(r_{2}\right)-H\left(r_{1}\right)+H\left(r_{2}\right) \\
= & \exp \left\{-\frac{r_{2}}{\mu_{X}}\right\}-\exp \left\{-\frac{r_{1}}{\mu_{X}}\right\}-H\left(r_{1}\right)+H\left(r_{2}\right) \\
= & \exp \left\{-\frac{r_{2}}{\mu_{X}}\right\}-R_{X}\left(r_{2}\right)+R_{X}\left(r_{1}\right)-\exp \left\{-\frac{r_{1}}{\mu_{X}}\right\},
\end{aligned}
$$

where $R_{X}$ denotes the survival function of $X$.

The result now follows from the fact that if $X$ is NBUE then, by Cheng and He (1988),

$$
\sup _{t>0}\left|R_{X}(t)-\exp \left\{-\frac{t}{\mu_{X}}\right\}\right| \leq 1-\exp \left\{-\sqrt{2 A_{X}}\right\},
$$

and if $X$ is NWUE then, by He and Cheng (1987),

$$
\sup _{t>0}\left|R_{X}(t)-\exp \left\{-\frac{t}{\mu_{X}}\right\}\right| \leq \sqrt{A_{X}^{2}+2 A_{X}}-A_{X} .
$$

This completes the proof.

Now, relations (28) and (29) in combination with (30), (31), and Lemma 4 yield the following error estimates (as a matter of fact, we use (16) and (17) due to the upper bounding of $\rho_{\mathrm{tv}}$ and $\rho_{\mathrm{k}}$, which lead to exactly the same results).

Proposition 2. Let $X_{1}, X_{2}, \ldots, X_{n}$ be i.i.d. observations from a distribution which is NBUE with mean $\mu_{X}$. Then, for $n \geq 4$,

$$
\begin{aligned}
& \rho_{\mathrm{TV}}\left(S_{n}^{X}, g\left(n, \frac{1}{\mu_{X}}\right)\right) \leq\left(8 B_{X}^{2}+2 h_{n} A_{X}+\frac{h_{n} A_{X}}{4 B_{X}}\right)\left(1+4 B_{X}\right)^{n-2}-\frac{h_{n} A_{X}}{4 B_{X}}, \\
& \rho_{\mathrm{K}}\left(S_{n}^{X}, g\left(n, \frac{1}{\mu_{X}}\right)\right) \leq\left(4 B_{X}^{2}+2 m_{n} A_{X}+\frac{m_{n} A_{X}}{4 B_{X}}\right)\left(1+4 B_{X}\right)^{n-2}-\frac{m_{n} A_{X}}{4 B_{X}},
\end{aligned}
$$

where $B_{X}:=1-\exp \left\{-\sqrt{2 A_{X}}\right\}$ and $A_{X}, h_{n}$, and $m_{n}$ are given by (25), (26), and (27), respectively.

Proposition 3. Let $X_{1}, X_{2}, \ldots, X_{n}$ be i.i.d. observations from a distribution which is NWUE with mean $\mu_{X}$. Then, for $n \geq 4$,

$$
\begin{aligned}
& \rho_{\mathrm{TV}}\left(S_{n}^{X}, g\left(n, \frac{1}{\mu_{X}}\right)\right) \leq\left(8 D_{X}^{2}+2 h_{n} A_{X}+\frac{h_{n} A_{X}}{4 D_{X}}\right)\left(1+4 D_{X}\right)^{n-2}-\frac{h_{n} A_{X}}{4 D_{X}}, \\
& \rho_{\mathrm{K}}\left(S_{n}^{X}, g\left(n, \frac{1}{\mu_{X}}\right)\right) \leq\left(4 D_{X}^{2}+2 m_{n} A_{X}+\frac{m_{n} A_{X}}{4 D_{X}}\right)\left(1+4 D_{X}\right)^{n-2}-\frac{m_{n} A_{X}}{4 D_{X}},
\end{aligned}
$$


where $D_{X}:=\left(A_{X}^{2}+A_{X}\right)^{1 / 2}-A_{X}$ and $A_{X}, h_{n}$, and $m_{n}$ are given by (25), (26), and (27), respectively.

The error estimates of Propositions 2 and 3 are useful both in theory and practice for fixed $n$, in cases where we cannot invoke central limit theory to approximate the distribution of the sum $S_{n}^{X}$ by the normal distribution.

Remark 5. As the coefficient of variation of $X$ tends to $1, A_{X}$ tends to 0 and, consequently, the upper bounds of Propositions 2 and 3 tend to 0 . This is reasonable, given that the coefficient of variation of an exponential random variable is equal to 1 .

Remark 6. Clearly, Propositions 2 and 3 provide bounds for the Weibull distribution considered in Subsection 3.1 with

$$
A_{X}=\left|1-\frac{\Gamma(1+2 / \alpha)}{2 \Gamma^{2}(1+1 / \alpha)}\right| .
$$

\subsection{Distance between the distribution of the sum of i.i.d. Student random variables and a normal distribution}

Assume that $T_{1}, T_{2}, \ldots, T_{n}$ are i.i.d. random variables following the Student $t$-distribution with $k$ degrees of freedom, $k>2$. In this case we have $\mu_{T}=0$ and $\sigma_{T}^{2}=k /(k-2)$. Furthermore, let $Z_{1}, Z_{2}, \ldots, Z_{n}$ be i.i.d. normally distributed random variables with $\mu_{Z}=0$ and $\sigma_{Z}^{2}=1$. Obviously, the sum $S_{n}^{Z}$ follows the normal distribution $N(0, n)$ and, consequently, $S_{n}^{Z} / \sqrt{n} \sim N(0,1)$. We also have $T_{i} \geq_{\mathrm{cx}} Z_{i}$ for all $i=1,2, \ldots, n$.

Let $f_{n-1}$ denote the density of $N(0, n-1)$. Clearly,

$$
\left|\sigma_{Z}^{2}-\sigma_{T}^{2}\right|=\frac{k}{k-2}-1=\frac{2}{k-2}, \quad k>2 .
$$

In addition, the norms $\left\|f_{n-1}^{(1)}\right\|_{\infty}$ and $\left\|f_{n-1}^{(2)}\right\|_{1}$ of the normal distribution $N(0, n-1)$ are given by

$$
\left\|f_{n-1}^{(1)}\right\|_{\infty}=\frac{1}{(n-1) \sqrt{2 \pi \mathrm{e}}} \quad \text { and } \quad\left\|f_{n-1}^{(2)}\right\|_{1}=\frac{4}{(n-1) \sqrt{2 \pi \mathrm{e}}} .
$$

Let $h$ and $\phi$ denote the densities of $T_{i}$ and $Z_{i}$, respectively. Then $h$ and $\phi$ cross each other at exactly two (symmetric) points, say $-r$ and $r$, since $T_{i} \geq_{\mathrm{cx}} Z_{i}$. Thus, it is easy to see that

$$
\begin{aligned}
\rho_{\mathrm{tv}} & =\rho_{\mathrm{TV}}\left(T_{i}, N(0,1)\right) \\
& =\frac{1}{2} \int_{-\infty}^{\infty}|h(t)-\phi(t)| \mathrm{d} t \\
& =\frac{1}{2} \int_{-\infty}^{-r}(h(t)-\phi(t)) \mathrm{d} t+\frac{1}{2} \int_{-r}^{r}(\phi(t)-h(t)) \mathrm{d} t+\frac{1}{2} \int_{r}^{\infty}(h(t)-\phi(t)) \mathrm{d} t \\
& =2\left(F_{T}(r)-\Phi(r)\right),
\end{aligned}
$$

where $F_{T}$ is the CDF of the Student $t$-distribution with $k$ degrees of freedom and $\Phi$ is the CDF of $N(0,1)$.

Here, it is important to note that Papadatos and Papathanasiou (1995, relation (5.2)) provided the following simple upper bound for the distance $\rho_{\mathrm{TV}}\left(T_{i}, N(0,1)\right)$ :

$$
\rho_{\mathrm{TV}}\left(T_{i}, N(0,1)\right) \leq \frac{4}{k-2},
$$


which establishes that the rate of convergence is at least $O(1 / k)$. Therefore, since $\rho_{\mathrm{K}}$ and $\rho_{\mathrm{TV}}$ are stable under scale changes and by taking into consideration (16), (18), Corollary 1, (32), and (33), we obtain the following error estimates.

Proposition 4. Let $T_{1}, T_{2}, \ldots, T_{n}$ be i.i.d. random variables which follow the Student $t$-distribution with $k$ degrees of freedom, $k>2$. For $n \geq 2$, we have

$$
\begin{aligned}
\rho_{\mathrm{TV}}\left(\frac{S_{n}^{T}}{\sqrt{n}}, N(0,1)\right) \leq & B_{\mathrm{TV}}^{\mathcal{N}} \\
:= & \left(\frac{32}{(k-2)^{2}}+\frac{1+16(k-2)^{-1}}{4(n-1) \sqrt{2 \pi e}}\right)\left(1+\frac{8}{k-2}\right)^{n-2} \\
& -\frac{1}{4(n-1) \sqrt{2 \pi \mathrm{e}}}, \\
\rho_{\mathrm{K}}\left(\frac{S_{n}^{T}}{\sqrt{n}}, N(0,1)\right) \leq & B_{\mathrm{K}}^{\mathcal{N}} \\
:= & \left(\frac{32}{(k-2)^{2}}+\frac{1+16(k-2)^{-1}}{8(n-1) \sqrt{2 \pi e}}\right)\left(1+\frac{8}{k-2}\right)^{n-2} \\
& -\frac{1}{8(n-1) \sqrt{2 \pi \mathrm{e}}} .
\end{aligned}
$$

We note that in Proposition 4 , for the distance $\rho_{\mathrm{K}}$, we used $\delta=\min \left\{\rho_{\mathrm{tv}}, 3 M^{2 / 3} \zeta^{1 / 3}\right\}$ with

$$
M=\sup _{y \in \mathbb{R}} f(y)=\sup _{z \in \mathbb{R}}\left(\frac{\exp \left\{-z^{2} / 2\right\}}{\sqrt{2 \pi}}\right)=\frac{1}{\sqrt{2 \pi}}
$$

(recall (8)). Thus, we obtain $\delta \leq \min \left\{4 /(k-2), 3 /(2 \pi(k-2))^{1 / 3}\right\}$ and the minimum is always equal to $4 /(k-2)$ for $k \geq 6$.

Theorem 2.1 of Cacoullos et al. (1997) combined with Theorem 2.1 of Cacoullos et al. (2001) yields the following upper bound for the total variation distance:

$$
\begin{aligned}
\rho_{\mathrm{TV}}\left(\frac{S_{n}^{T}}{\sqrt{n}}, N(0,1)\right) & \leq B_{\mathrm{CPP}} \\
& :=\frac{3}{\sqrt{2 n(k-1)(k-4)}}+\frac{4}{k-2} \\
& \sim\left(\frac{2.12132}{\sqrt{n}}+4\right) \frac{1}{k}
\end{aligned}
$$

for fixed $n$ and $k \rightarrow \infty$ (the notation $a_{k} \sim b_{k}$ implies that $\lim _{k \rightarrow \infty}\left(a_{k} / b_{k}\right)=1$ ). The bounds $B_{\mathrm{TV}}^{\mathcal{N}}$ and $B_{\mathrm{K}}^{\mathcal{N}}$ provided by Proposition 4 are asymptotically equal to

$$
\begin{aligned}
B_{\mathrm{TV}}^{\mathcal{N}} & \sim \frac{2 n}{(n-1) \sqrt{2 \pi \mathrm{e}}} \frac{1}{k}, \\
B_{\mathrm{K}}^{\mathcal{N}} & \sim \frac{n}{(n-1) \sqrt{2 \pi \mathrm{e}}} \frac{1}{k},
\end{aligned}
$$

for fixed $n$ and $k \rightarrow \infty$. Both error estimates (35) and (36) are of order $O(1 / k)$, but, asymptotically, (36) is obviously smaller than (35) due to the existence of the constant $\sqrt{2 \pi \mathrm{e}} \simeq$ 4.13273 in the denominator of (36). 
TABLE 2.

\begin{tabular}{ccccccccc}
\hline \multicolumn{3}{c}{$n=3$} & \multicolumn{4}{c}{$n=5$} \\
\hline$k$ & $B_{\mathrm{CPP}}$ & $B_{\mathrm{TV}}^{\mathcal{N}}$ & $k$ & $B_{\mathrm{CPP}}$ & $B_{\mathrm{TV}}^{\mathcal{N}}$ & $k$ & $B_{\mathrm{CPP}}$ & $B_{\mathrm{TV}}^{\mathcal{N}}$ \\
\hline 25 & 0.228468 & 0.120412 & 30 & 0.177406 & 0.122136 & 35 & 0.141875 & 0.216775 \\
30 & 0.187460 & 0.083342 & 35 & 0.150434 & 0.084298 & 40 & 0.123166 & 0.139503 \\
35 & 0.158937 & 0.062061 & 40 & 0.130582 & 0.062309 & 45 & 0.108817 & 0.097159 \\
40 & 0.137949 & 0.048610 & 45 & 0.115359 & 0.048372 & 50 & 0.097463 & 0.071707 \\
45 & 0.121859 & 0.039502 & 50 & 0.103316 & 0.038952 & 55 & 0.088254 & 0.055300 \\
50 & 0.109130 & 0.033007 & 55 & 0.093549 & 0.032263 & 60 & 0.080636 & 0.044132 \\
55 & 0.098810 & 0.028186 & 60 & 0.085470 & 0.027325 & 65 & 0.074228 & 0.036191 \\
60 & 0.090273 & 0.024491 & 65 & 0.078675 & 0.023562 & 70 & 0.068764 & 0.030342 \\
65 & 0.083094 & 0.021584 & 70 & 0.072882 & 0.020620 & 80 & 0.059939 & 0.022455 \\
70 & 0.076972 & 0.019247 & 80 & 0.063525 & 0.016355 & 90 & 0.053122 & 0.017501 \\
\hline
\end{tabular}

In Table 2, numerical comparisons between the bounds $B_{\mathrm{TV}}^{\mathcal{N}}$ of Proposition 4 and $B_{\mathrm{CPP}}$ of (34) are presented for several values of $k$ and $n$. Obviously, for fixed $n$, both error estimates tend to 0 when $k \rightarrow \infty$, as expected. The bounds were computed using MATHEMATICA software. Here, it is important to note that, for the calculations, we used the exact values of the bounds given by Proposition 4 and (34), and not the asymptotic error estimates (35) and (36).

\section{Acknowledgement}

The authors are grateful to the anonymous referee, whose suggestions led to a much improved version of the manuscript.

\section{References}

Barlow, R. E. And Proschan, F. (1975). Statistical Theory of Reliability and Life Testing. Holt, Rinehart and Winston, New York.

Boutsikas, M. V. and Vaggelatou, E. (2008). A new method for obtaining sharp compound Poisson approximation error estimates for sums of locally dependent random variables. Preprint.

Cacoullos, T., Papadatos, N. and Papathanasiou, V. (1997). Variance inequalities for covariance kernels and applications to central limit theorems. Theory Prob. Appl. 42, 195-201.

Cacoullos, T., Papadatos, N. and Papathanasiou, V. (2001). An application of a density transform and the local limit theorem. Theory Prob. Appl. 46, 803-810.

Cheng, K. And He, Z. F. (1988). Exponential approximations in the classes of life distributions. Acta Math. Appl. Sinica (English Ser.) 4, 234-244.

Daley, D. J. (1988). Tight bounds on the exponential approximation of some aging distributions. Ann. Prob. 16, 414-423.

He, Z. AND Cheng, K. (1987). Exponential approximations for some classes of life distributions. In Reliability Theory and Applications, eds S. Osaki et al., World Scientific, Singapore, pp. 113-126.

KAAS, R. (1993). How to and how not to compute stop-loss premiums in practice. Insurance Math. Ecomom. 13, $241-254$.

Müller, A. And Stoyan, D. (2002). Comparison Methods for Stochastic Models and Risks. John Wiley, Chichester.

Papadatos, N. and Papathanasiou, V. (1995). Distance in variation between two arbitrary distributions via the associated $w$-functions. Theory Prob. Appl. 40, 685-694.

Rachev, S. T. (1991). Probability Metrics and the Stability of Stochastic Models. John Wiley, Chichester.

RaCHEv, S. T. AND RÜSCHENDORF, L. (1990). Approximation of sums by compound Poisson distributions with respect to stop-loss distances. Adv. Appl. Prob. 22, 350-374. 
Shaked, M. And Shanthikumar, J. G. (2007). Stochastic Orders. Springer, New York.

SzekLI, R. (1995). Stochastic Ordering and Dependence in Applied Probability (Lecture Notes Statist. 97). Springer, New York.

VaGgelatou, E. (2009). A new method for bounding the distance between sums of independent integer-valued random variables. To appear in Methodology Comput. Appl. Prob.

Zolotarev, V. M. (1983). Probability metrics. Theory Prob. Appl. 28, 278-302. 